\title{
Author Correction: Configurable phonon polaritons in twisted $\boldsymbol{\alpha}-\mathrm{MoO}_{3}$
}

Mingyuan Chen, Xiao Lin (1), Thao H. Dinh, Zhiren Zheng, Jialiang Shen, Qiong Ma, Hongsheng Chen (D), Pablo Jarillo-Herrero (iD and Siyuan Dai (iD

Correction to: Nature Materials https://doi.org/10.1038/s41563-020-0732-6, published online 13 July 2020.

In the version of this Article originally published, the following sentence was missing from the Acknowledgements section: 'Work at Auburn University was supported by the Auburn University Intramural Grants Program and the National Science Foundation under grant no. DMR-2005194. It should have followed the first sentence of the Acknowledgements. This has now been corrected in all versions of the Article.

Published online: 28 July 2020

https://doi.org/10.1038/s41563-020-0781-x

(C) The Author(s), under exclusive licence to Springer Nature Limited 2020 